\title{
Peut-on vivre avec le ragondin ? Les représentations sociales reliées à un animal envahissant
}

\author{
Catherine Mougenot ${ }^{\mathrm{a}}$, Laurence Roussel ${ }^{\mathrm{b}}$ \\ a Sociologue, SEED/Département Sciences et gestion de l'environnement, Université de Liège, Bâtiment BE FUL, \\ 185 avenue de Longwy, 6700 Arlon, Belgique \\ b Écologue, SEED/Département Sciences et gestion de l'environnement, Université de Liège, Bâtiment BE FUL, \\ 185 avenue de Longwy, 6700 Arlon, Belgique
}

\author{
Mots-clés : \\ ragondin; \\ espèce invasive ; \\ représentations \\ sociales
}

\section{Keywords:}

coypu;

invasive species;

social representations

\begin{abstract}
Résumé - Aujourd'hui les introductions d'espèces exotiques, souvent qualifiées d'espèces invasives, sont vues comme une menace pour les écosystèmes. En menant une enquête sur les représentations liées au ragondin, introduit en Europe depuis près de cent ans, nous avons découvert que cet animal a aussi une nature domestique, sauvage et nuisible. De notre étude, il ressort également que ces représentations sont liées aux actions menées contre lui. En effet elles supposent des formes d'organisation et des choix techniques qui ont des impacts sur la nature et les hommes eux-mêmes. De là découle qu'il n'y a pas de clivages simples entre humains et espèces animales, mais de nombreuses lignes de partage, fortement contextualisées, inscrites dans des lieux et des moments. Les représentations de la nature sont aussi liées à celles des territoires, aux risques sanitaires acceptables et au profil des acteurs qui doivent mener les actions de lutte. On comprend alors qu'il est bien difficile aujourd'hui d'aligner tous ces points de manière satisfaisante.
\end{abstract}

\begin{abstract}
Can we live with the Coypu? Social representations of an invasive animal. It has by now been demonstrated that introducing non-indigenous (exotic) species can threaten ecosystems, but how are these species perceived? This article considers a specific case, the coypu, introduced into Europe over one hundred years ago for trade in its fur. From our survey, carried out among 79 people (managers of natural areas, agricultural producers, scientists and decision-makers), it is evident that this animal has a special story because it has been transformed and, at the same time, has been attributed "many natures", that is, it has been given many representations. The coypu is perceived both as a domestic and as a wild animal, as well as an exotic animal and as a pest. Nevertheless, in France, the law first and foremost defines the animal as a pest, which gives good reason for the two principal means of trying to control it: poisoning and trapping. However, such methods presuppose forms of organization and technical choices that have an impact on nature and on humans themselves, and are thus debated and sometimes challenged. This explains why, in the present study, representations of this animal are also systematically connected to the actions taken to control it. In the words of the people questioned, place is being sought that should be given - or denied - this animal. The comments thus emphasize the many choices that remain to be made because they depend on questions that are persistently open-ended and overlapping. New categories of representations piece together the different natures of the coypu. From this it follows that there is no simple cleavage between humans and animal species, but many shared traits, clearly dependent on context, including time and place. Representations of nature are also connected to representations of the territories, to acceptable health risks (the coypu is in fact a leptospirosis carrier) and to the profile of those who should lead the fight. In addition, it is understood that it is very difficult to coordinate these different elements in a satisfactory manner.
\end{abstract}

Le ragondin (Myocastor coypu) a un corps de castor, une queue-de-rat, des pattes de canard, il plonge comme une loutre et mange comme un écureuil... (Maurice, 1931).

Auteur correspondant : cmougenot@ulg.ac.be
S'agit-il là des raisons pour lesquelles il apparaît intraitable? Difficile à classer, à gérer, ou même à étudier? Un tel animal ne peut sans doute que réserver des surprises, susciter de l'ambivalence, brouiller les clivages habituels... 
Dans cet article nous montrerons que le ragondin a une histoire particulière qui voit se succéder et en même temps se superposer "plusieurs natures », c'est-à-dire plusieurs façons de le voir et d'interagir avec lui. Ensuite, nous découvrirons que lorsqu'on cherche à définir la place qu'il faudrait lui accorder - ou refuser -, les choix qui restent à faire suscitent de nombreuses questions, toujours ouvertes et imbriquées les unes dans les autres. Ce sont là de nouvelles catégories qui recomposent autrement encore les différentes natures du ragondin.

Ce texte est issu d'une recherche portant sur «les perceptions du caractère invasif attribuées au ragondin et au rat musqué ». Un travail qui s'est déroulé entre 2001 et $2003^{1}$, construit à partir d'entretiens qualitatifs réalisés au cours de quatre missions réalisées dans des zones dites "infestées ", à savoir le Marais poitevin, la Camargue, les pays de Loire et la Bretagne, et le Nord (pour le rat musqué). Nous y avons rencontré des membres de groupes techniques agricoles, des gestionnaires d'espaces naturels, des responsables administratifs et politiques, des chasseurs, piégeurs et exploitants agricoles (67 personnes) directement concernés par les actions de lutte contre ces animaux. Cette enquête a été complétée par des entretiens avec des responsables administratifs ou membres d'associations naturalistes au niveau national et des scientifiques directement concernés par l'étude des rongeurs (12 personnes). Avec tous ces interlocuteurs, nous avons eu des conversations longues, très peu directives et qui se sont parfois déroulées sur « leur » terrain où nous les avons volontiers accompagnés, notre objectif étant, avant tout, de collecter leurs « histoires » (Mougenot, 2003) à propos de ces animaux, des luttes menées contre eux et des politiques qui réglementent leur gestion.

\section{Un animal qui n'a pas de nature définitive}

«Le ragondin est un animal inoffensif qui cause des dégâts considérables » (un piégeur).

En mobilisant les différents éléments apportés par les personnes rencontrées, nous avons cherché à reconstituer la suite des événements qui marquent la présence du ragondin en France. Mais c'est une chronologie "étrange » qui est présentée ici, parce qu'elle avait d'abord pour but de construire l'espace des représentations liées à cet animal. Il s'agissait donc moins de chercher une correspondance avec des faits réellement avérés que de mettre en évidence comment différentes catégories de représentation ont pu s'ajouter les unes aux autres, prendre des significations différentes au cours du temps, et finalement produire l'imbroglio dans lequel cet animal semble

\footnotetext{
${ }^{1}$ Cette recherche fait partie du programme portant sur les invasions biologiques (INVABIO) financé par le ministère de l'Écologie et du Développement durable.
}

pris aujourd'hui. Cela dit, en dépit du fait que certains de nos interlocuteurs ont parfois évoqué des épisodes qui n'étaient pas totalement concordants, cette chronologie suit, globalement, les données réunies par F. Léger et P. Migot (Encadré 1).

\section{Une nature exotique}

Le ragondin a été importé en Europe pour le commerce de sa peau. Il est pourtant nécessaire de signaler qu'il a d'abord été introduit dans nos pays, dès le milieu du XIX ${ }^{e}$ siècle par quelques membres d'une société d'acclimatation. Pour ces grands voyageurs passionnés d'exotisme, les animaux venus d'ailleurs étaient intéressants à divers titres : pour l'étude, mais aussi pour la diversification des gibiers chassables, ou encore pour la production de viandes fines. En ce qui concerne le ragondin, ce caractère "exotique » a encore été amplifié par le fait qu'il était régulièrement exposé dans les foires. Il y était montré comme un animal maléfique, un gros rat capable de dévorer les enfants désobéissants.

Près de cent ans plus tard, le qualificatif d'exotique est devenu un synonyme de «non indigène » ou même $\mathrm{d}^{\prime}$ « invasif ». Les scientifiques démontrent pourquoi et comment les déplacements d'espèces représentent une menace pour les écosystèmes, en soulignant que, lorsque celles-ci deviennent proliférantes, elles constituent la deuxième cause de perte de la biodiversité. Dans le cas du ragondin, il n'est pas explicitement démontré qu'il exerce une concurrence sur des espèces menacées. En revanche, plusieurs gestionnaires nous signalent que dans certains espaces naturels, il est susceptible de perturber les systèmes hydrauliques et de convoiter la même nourriture que d'autres animaux protégés.

\section{Une nature domestique}

La deuxième étape qui marque la présence du ragondin en Europe est ensuite clairement celle de l'élevage qui se développe dans les années 1920. Suite aux premières expériences menées dans leur région d'origine (Chili, Argentine), certaines sous-espèces sont sélectionnées pour leur prolificité. Le ragondin devient alors un animal «domestique », élevé et reproduit en captivité dans un but de rentabilité. En France, ces animaux sont reproduits en cage ou dans des parcs, d'abord pour la vente de leur peau (pelleterie), de leurs poils (chapellerie) et ensuite pour leur viande. Les élevages sont très diversement répartis, ce qui provoquera plus tard des foyers de colonisation disséminés sur l'ensemble du territoire.

L'élevage « rationnel » du ragondin va connaître des hauts et des bas. En grande difficulté suite à la crise des années 1930, il va redémarrer ensuite pour s'éteindre progressivement avec la faillite du commerce des peaux en Europe occidentale, à partir des années 1960. Actuellement, la pelleterie est une activité qui survit de façon 
Encadré 1. Quelques jalons sur l'histoire du ragondin en France (F. Léger et P. Migot, inédit)

\begin{tabular}{|c|c|}
\hline Période & Situation \\
\hline 1782 & $\begin{array}{l}\text { Le ragondin n'a été connu que très tard des naturalistes de nos pays. Il a été décrit pour la première fois par deux } \\
\text { voyageurs. En premier lieu l'ecclésiastique chilien Molina qui le fait connaître en } 1782 \text { dans son « Histoire naturelle } \\
\text { du Chili » sous son appellation sud américaine de Coypou. La description fournie par Molina est encore laconique } \\
\text { et il parle d'une espèce de rat d'eau de la grosseur et de la couleur d'une loutre. }\end{array}$ \\
\hline 1801 & $\begin{array}{l}\text { Une nouvelle description est fournie par Félix de Azara dans son ouvrage "Essais sur l'Histoire naturelle des } \\
\text { quadrupèdes de la Province du Paraguay » publié à Paris et qui évoque l'animal comme une nouvelle espèce de cabiai } \\
\text { sous la dénomination de Quouyia. }\end{array}$ \\
\hline 1805 & $\begin{array}{l}\text { La science se laisse devancer par le commerce... Pendant une dizaine d'année, la fourrure de ce rongeur, introduite } \\
\text { dans le commerce européen, sous le nom de racoonda, resta inconnue des naturalistes. En 1805, Étienne Geoffroy } \\
\text { Saint-Hilaire publie sur ce rongeur après avoir examiné, dans un riche magasin de fourrures à Paris, un nombre } \\
\text { considérable de peaux qu'il attribue sans ambiguïté à cet animal. La fourrure du ragondin est alors principale- } \\
\text { ment employée en chapellerie pour la fabrication du feutre. Étienne Geoffroy Saint-Hilaire exhume également } \\
\text { au Muséum national d’histoire naturelle, parmi les manuscrits du célèbre naturaliste Philibert Commerson, un } \\
\text { croquis de l'espèce qui lui avait été remis par Bougainville. Commerson avait donné provisoirement le nom de } \\
\text { Myopotamus bonariensis à cette espèce. }\end{array}$ \\
\hline 1830 & $\begin{array}{l}\text { On s'intéresse à l'anatomie du ragondin... Isidore Geoffroy Saint-Hilaire signale déjà en } 1830 \text { que l'une des particu- } \\
\text { larités anatomiques de l'espèce réside dans la position dorsolatérale remarquable des mamelles chez la femelle. Son } \\
\text { observation passa inaperçue tout comme celle qui fut faite, quelques années plus tard par M. Christy de Londres. } \\
\text { La position exceptionnelle des tétines va faire l'objet de communications presque simultanément aux Académies } \\
\text { de Stockholm, de Copenhague et de Bruxelles une dizaine d'années plus tard. En France, M. Lereboullet, professeur } \\
\text { de zoologie et d'anatomie comparée à la faculté des sciences de Strasbourg va réaliser un travail approfondi sur } \\
\text { l'anatomie de l'espèce. }\end{array}$ \\
\hline 1858 & $\begin{array}{l}\text { A Paris, le Jardin des Plantes possède alors plusieurs couples de ragondins. Les parisiens, à l'aquarium du boulevard } \\
\text { Montmartre, pouvaient également faire connaissance avec l'un de ces rongeurs qui était d'une grande familiarité } \\
\text { et accourait lorsqu'on prononçait son nom... }\end{array}$ \\
\hline Vers 1882 & $\begin{array}{l}\text { M.G. Pays Mellier, naturaliste et propriétaire du parc zoologique de la Pataudière à Champigny-sur-Veude se } \\
\text { procure deux couples de ragondins achetés jeunes à Anvers et Rotterdam et les installe dans son parc où il obtient } \\
\text { la reproduction. Il publie ses observations dans le bulletin de la Société nationale d'acclimatation de France. } \\
\text { D'autres propriétaires et amateurs éclairés font de même et le ragondin fait l'objet d'échanges. Il est alors perçu } \\
\text { comme un animal d'ornement et de curiosité digne d'intérêt et encore méconnu. Contrairement à ce qui a été } \\
\text { affirmé dans la littérature, ces expériences ont été menées par des hommes nullement motivés par l'élevage du } \\
\text { ragondin dans un but commercial pour la fourrure. }\end{array}$ \\
\hline $\begin{array}{l}\text { Début du } \\
\text { XXe siècle }\end{array}$ & $\begin{array}{l}\text { Avant la première guerre mondiale, on découvre avec curiosité ce gros rongeur impressionnant et certains profitent } \\
\text { de la crédulité et de l'ignorance des gens. Ainsi, des ragondins sont exhibés dans les foires, décrits comme des } \\
\text { soi-disant "rats monstres » ayant dévoré des enfants vivants... ou comme des "rats géants ", capturés dans les } \\
\text { égouts de Lyon ou autres grandes villes. }\end{array}$ \\
\hline 1918 & $\begin{array}{l}\text { C. Kretzschmar, le célèbre fourreur de Chalon-sur-Saône indique en } 1918 \text { au sujet du ragondin : «Il est commun } \\
\text { aujourd'hui dans nos jardins zoologiques et se reproduit facilement en captivité. » }\end{array}$ \\
\hline Années 1920 & $\begin{array}{l}\text { En Argentine, l'élevage du ragondin est encouragé par le ministère de l'Agriculture de ce pays en vue de limiter } \\
\text { la chasse intensive dont l'espèce était l'objet et satisfaire la demande grandissante sur les peaux de ragondins. }\end{array}$ \\
\hline 1927 & $\begin{array}{l}\text { Les débuts en France de l'élevage commercial du ragondin pour la fourrure... À quelques mois d'intervalle, deux } \\
\text { fermes d'élevages d'animaux à fourrure installés dans le Lot-et-Garonne et dans le Finistère réalisent les premiers } \\
\text { essais d'élevage en captivité du ragondin en France dans des perspectives commerciales pour la pelleterie. }\end{array}$ \\
\hline 1928 & $\begin{array}{l}\text { Les premiers sujets nés en France dans les élevages pelletiers sont présentés à la grande exposition internationale } \\
\text { d'aviculture de Paris en février } 1928 .\end{array}$ \\
\hline $\begin{array}{l}\text { Fin des } \\
\text { années } 1920 \\
\text { et début des } \\
\text { années } 1930\end{array}$ & $\begin{array}{l}\text { L'élevage se développe en France mais pas uniquement et la demande de reproducteurs adultes est très active en } \\
\text { Europe, notamment en direction de l'Allemagne. Au cours des années 1930, le monde de l'élevage du ragondin en } \\
\text { France est très contrasté. La profession se partage entre une dizaine d'élevages digne de ce nom et une multitude } \\
\text { de petits élevages amateurs qui ne possèdent que quelques couples et débutent souvent avec un couple. Ces petits } \\
\text { élevages au nombre de } 200 \text { environ sont tenus par des exploitants agricoles, des aviculteurs, des retraités, etc. et } \\
\text { offrent un appoint de revenus. }\end{array}$ \\
\hline 1930 & $\begin{array}{l}\text { Pour défendre l'élevage de l'espèce, une association va se créer sous le nom de « Nutria-Club » et le sous-titre } \\
\text { suivant : «Association française des éleveurs de ragondins ». Cette association est une section autonome de la } \\
\text { fédération des associations françaises et des éleveurs d'animaux à fourrure. }\end{array}$ \\
\hline $\begin{array}{l}\text { Début des } \\
\text { années } 1930\end{array}$ & $\begin{array}{l}\text { Le docteur Albert Maurice mène des essais sur l'élevage du ragondin en semi-liberté en Sologne dans son élevage } \\
\text { et sur les étangs adjacents à Yvoy-le-Marron (Loir et Cher). Il propose d'utiliser le ragondin dans le faucardage des } \\
\text { étangs et fait la promotion de l'espèce comme nouveau gibier de chasse. Il publie un ouvrage de référence. }\end{array}$ \\
\hline
\end{tabular}




\begin{tabular}{|c|c|}
\hline Années 1930 & $\begin{array}{l}\text { Des acclimatations en nature sont signalées. Une polémique fait rage en Sologne entre le docteur Albert Maurice } \\
\text { et un propriétaire d'étangs qui va interpeller les pouvoirs publics. Ceux-ci engagent dès 1932, une réflexion sur le } \\
\text { thème du ragondin et pour éclairer le débat confie des travaux à A. Chappellier, directeur du service des vertébrés } \\
\text { au Centre national de recherches agronomiques de Versailles ainsi qu'à E. Bourdelle, du Muséum national d'histoire } \\
\text { naturelle et A. Henry de l'École nationale vétérinaire de Maisons-Alfort. }\end{array}$ \\
\hline 1937 & Le ministère de l'Agriculture donne au ragondin le statut d'espèce nuisible. \\
\hline 1939 & L'approche de la guerre et la mobilisation vont marquer un coup d'arrêt de l'élevage du ragondin en France. \\
\hline Années 1950 & $\begin{array}{l}\text { Le renouveau de l'élevage du ragondin avec une seconde génération d'éleveurs... } \\
\text { Les années } 1954 \text { à } 1956 \text { connaissent un développement rapide des élevages dans les départements de l'Eure tant } \\
\text { sur les bords de l'Eure et de l'Iton que dans des exploitations alimentées par l'eau de distribution communale. On } \\
\text { signale des élevages sur l'Yonne et ses affluents, en Bretagne, dans le Pays basque, la région parisienne, la vallée } \\
\text { de la Somme, le Morvan, la Sologne, La Dombes, la Camargue. Dans l'ensemble, la moitié sud du pays comporte } \\
\text { peu d'élevages ainsi que les régions de l'Est, jugées trop froides. A l'époque, la région d'élection des élevages est } \\
\text { située au nord de la Loire, dans des contrées à climat atlantique ou semi-continental. On estime qu'il y avait dans } \\
\text { les élevages français, en 1956, près de } 7000 \text { reproducteurs. }\end{array}$ \\
\hline Années 1960 & $\begin{array}{l}\text { L'espèce débute son acclimatation à grande échelle en nature en France... } \\
\text { Avec le développement de l'élevage pelletier du ragondin qui se poursuit au cours des années 1960, de nombreux } \\
\text { facteurs vont favoriser son apparition et son développement en nature. En premier lieu, l'importance des effectifs } \\
\text { nécessaires à l'élevage donne l'occasion d'évasions plus fréquentes, souvent répétées avec des effectifs pouvant } \\
\text { laisser espérer une acclimatation durable en nature. En outre, la large diffusion de l'élevage sur de vastes étendues } \\
\text { géographiques et leur implantation, pour des raisons techniques liées à l'élevage de cette espèce, à proximité des } \\
\text { cours d'eau vont également favoriser son apparition dans les milieux naturels. Au cours des années 1960, l'espèce, } \\
\text { présente déjà des noyaux de dispersion actifs localisés au cours inférieur de la Garonne, aux zones humides des } \\
\text { Landes, au bassin de la Loire, au cours inférieur du Rhône mais aussi au bassin de la Seine dans les départements } \\
\text { de l'Aube et de la Marne. }\end{array}$ \\
\hline Années 1970 & Le ragondin se développe en nature et poursuit son extension. \\
\hline $\begin{array}{l}\text { Milieu des } \\
\text { années } 1980\end{array}$ & $\begin{array}{l}\text { Crise dans les professions de la fourrure. L'élevage pelletier du ragondin est en plein déclin et la grande majorité } \\
\text { des élevages cessent leurs activités. }\end{array}$ \\
\hline $\begin{array}{l}\text { Années } \\
1980-1990\end{array}$ & Expansion de l'espèce en France. Lutte intensive contre le ragondin (piégeage, empoisonnement). \\
\hline $2000-2005$ & e se poursuit avec suppression annoncée de la bromadiolone... \\
\hline
\end{tabular}

anecdotique (les peaux sont vendues pour un prix d'environ $5 \mathrm{FF}$ ) et la production de pâtés est également pratiquée en quelques endroits ${ }^{2}$ (dans ce cas, il s'agit exclusivement de produits d'élevage en raison des normes sanitaires en vigueur.)

Mais la nature domestique du ragondin s'exprime autrement encore, s'étant enrichie de la définition d'un « animal de compagnie ». Il nous est dit en effet, que les enfants des éleveurs avaient pour habitude d'avoir un ragondin apprivoisé à la maison, une tradition qui s'est maintenue ensuite chez certains piégeurs et qui existe encore aujourd'hui, même si c'est de façon marginale. Le ragondin est alors décrit comme un animal intelligent, "très sympathique ", voire même comme une "espèce de gros nounours » (au contraire du rat musqué qui, lui, est qualifié de «sournois»).

\section{Une nature sauvage}

Dès les années 1930, le ragondin commence à être relâché en pleine nature, un processus qui va s'accélérer avec la faillite des élevages. C'est un rongeur inféodé aux zones humides, et on découvre qu'il peut être très utile pour le faucardage (action de faucher les herbes

\footnotetext{
${ }^{2}$ Le ragondin est alors surnommé pour la cause «lièvre des marais ».
}

dans les rivières et canaux). En France, l'idée en revient au docteur Maurice qui réalise en Sologne ses propres observations dans les limites étroites d'étangs grillagés. Sur cette base, il développe une conception très positive du ragondin qu'il décrit comme un animal "plurifonctionnel » : utile pour la gestion des étangs puisqu'il a un impact considérable sur la diminution de la végétation, mais aussi pour le commerce de sa peau. De domestique, la nature du ragondin redevient donc progressivement «sauvage ». Dans la nature, ses populations se développeront d'abord d'une façon assez silencieuse. Il nous est rapporté que les naturalistes verront d'abord d'un assez bon œil cet animal entretenant spontanément rivières, étangs et canaux. Son image de «bon petit faucardeur » survivra ainsi un certain temps à la faillite des élevages.

Aujourd'hui, pour certains de nos interlocuteurs, la représentation de la nature sauvage du ragondin se combine surtout avec celle d'un animal vivant de façon autonome. Mais il est aussi «sociable» (il prend soin de ses petits) et surtout très «visible », ce qui explique la mise en avant d'un point de vue explicitement pédagogique : il est présenté comme faisant partie de la faune des zones humides (à travers des affiches, livrets, collections d'animaux empaillés...) et dans certains espaces protégés, on peut le voir évoluer en compagnie d'animaux indigènes, en particulier avec des castors. On peut aussi l'observer 


\begin{abstract}
Encadré 2. En Angleterre, une opération d'éradication réussie
«On devrait pouvoir évaluer le bonheur qu'ont éprouvé les Anglais, après avoir été débarrassés du ragondin! (un naturaliste).

En Europe, l'Angleterre représente un cas très particulier, puisque la population de ragondins y a été complètement éradiquée au cours des années 1980. Ce succès a marqué les esprits et, en France, beaucoup de personnes y font encore référence, notamment parce qu'il a été obtenu uniquement par piégeage.

Ce cas est sans doute exemplaire, mais pour deux raisons, au moins. D'abord parce qu'au début des interventions, l'effectif des animaux était véritablement faible : de 200000 environ dans les années 1970, il était retombé à 5000, après une période de grand froid. Ensuite, ce cas est représentatif d'une intégration réussie entre biologie appliquée et gestion des populations (Gosling, 1989) et entre les différents points de vue : technique, administratif et financier (Sheail, 2003).

M. Gosling, responsable du COYPU Research Laboratory a cherché à répondre à une double question : combien de piégeurs sont-ils nécessaires pour éradiquer ces rongeurs et, combien de temps cela prendra-t-il? Une stratégie qui a mis plus de 20 ans à aboutir, a été construite sur la base d'informations provenant d'agriculteurs, de gestionnaires de l'eau, de naturalistes et des expériences menées sur des bassins versants de taille réduite. Le succès remporté par Gosling souligne l'importance d'une intervention précoce (immédiatement après un hiver rigoureux) et de l'engagement de piégeurs nombreux et financièrement motivés.
\end{abstract}

dans quelques fermes pédagogiques, ou tout simplement dans les cours d'eau et canaux à proximité des villes. Le ragondin est alors décrit comme un excellent moyen pour faire découvrir la nature sauvage, en particulier aux enfants.

\section{Une nature nuisible}

Dans les années 1970 et en l'absence de tout prédateur (l'alligator et le jaguar ne font en effet pas partie de la faune locale), le ragondin colonise rapidement l'ouest et le sud de la France. On remarque alors qu'il est sensible aux grands froids et que quelques semaines de gel sévère peuvent réduire très fortement ses populations. Mais ses observateurs notent aussi que celles-ci se reconstituent ensuite très rapidement (Encadré 2).

Végétarien, le ragondin grignote volontiers les cultures qui avoisinent ses terriers. En creusant, il provoque des dégâts aux berges des rivières et canaux, ce qui nécessite un entretien très fréquent. Dans ce contexte, la lutte contre le ragondin, une espèce qui peut être chassée et classée animal «nuisible» (un statut légal qui impose l'obligation de le détruire sous certaines conditions) s'intensifie. Il peut donc être chassé, piégé, mais aussi empoisonné avec de la bromadiolone, un anticoagulant qui provoque des hémorragies internes ${ }^{3}$. Le ragondin est désormais perçu comme «multitude », et la prolificité qui avait justifié son introduction s'inverse, faisant de lui un «fléau ». Les dégâts qu'il provoque, ainsi que l'incapacité à les gérer ou à les prévoir exaspèrent. Du coup, son caractère d'animal visible se retourne également contre lui et les nombreux cadavres écrasés sur les routes deviennent alors les indicateurs d'une catastrophe non maîtrisée.

\footnotetext{
${ }^{3}$ Le statut juridique de l'espèce et les modalités de lutte contre l'espèce en France s'appuient sur des dispositions réglementaires prises respectivement en application du code de l'Environnement (articles L.424.1, L.427-8, R427-6 et 427-8) et du code rural (article L.251-3 et D.251-1). Certains articles ont des fondements anciens dont la loi du 3 mai 1844 sur la chasse, modifiée à plusieurs reprises. Dans ce cadre, l'espèce avait été déclarée «nuisible» dès 1937 (Chaigneau, 1967).
}

Dans notre étude, le projet initial était d'étudier les représentations d'un animal envahissant. Mais à travers l'histoire de la présence du ragondin en Europe, nous l'avons successivement découvert comme exotique, domestique, sauvage, nuisible... Les propriétés du ragondin telles qu'elles sont décrites, nous apparaissent alors aussi liées à l'espace, aux évolutions socioéconomiques, aux transformations des villes et campagnes. Des propriétés qui ne sont donc pas que physiques mais qui expriment aussi toute la gamme des relations que les humains construisent avec lui et qui expriment des rencontres successives, à travers lesquelles la nature change, mais les humains aussi (Melard, 2001 ; Despret, 2002).

Pour finir, nous retiendrons aussi de cette première partie que la représentation du ragondin comme espèce nuisible va s'imposer à toutes les autres, puisque c'est la seule qui a été véritablement consolidée par la loi ${ }^{4}$. Autrement dit, les « autres » natures de cet animal ne s'expriment actuellement que de façon marginale ou n'ont pas droit de cité. Pourtant la prédominance d'une nature nuisible n'est elle-même pas sans ambiguïté parce qu'elle évoque à la fois la catégorie ancienne (moyenâgeuse) d'un animal "mangeur de poules » et la représentation agronomique (moderne) d'un « organisme ravageur » des cultures. Et nous allons découvrir que cette dualité va être à l'origine d'un nouveau problème, c'est-àdire d'un débat portant sur le choix entre lutte mécanique et lutte chimique.

\section{Des questions qui n'ont pas de réponses définitives}

En France, quiconque respecte la législation relative au piégeage peut capturer et tuer des ragondins. En revanche, seuls les membres des fédérations départementales des groupements de défense contre les ennemis des cultures (FDGDEC) sont habilités à distribuer les carottes empoisonnées à la bromadiolone, des appâts qui

\footnotetext{
4 Bien entendu, la loi Barnier (1995) sur les espèces invasives peut également s'appliquer au ragondin, mais sans réelles implications pratiques.
} 


\section{Encadré 3. En France, une succession d'études et une mosaïque de partenaires}

En France, dès les années 1980, des chercheurs ont uni leurs voix pour dénoncer l'utilisation de la bromadiolone et proposer l'usage du piège comme alternative crédible. En 1983, un groupe de travail est mis en place à l'initiative du préfet de la région de Poitou Charente. Il réunit notamment : le centre CNRS de Chizé, le PNR du Marais poitevin, les services régionaux de la protection des végétaux, les fédérations départementales des groupements de défense contre les ennemis des cultures, le MNHN de Paris, l'INRA, l'École nationale vétérinaire de Nantes et de Lyon, l'Association de coordination de technique agricole (ACTA), des groupes de naturalistes, de piégeurs, etc. Ce groupe fonctionnera durant 5 ans, avec pour objectif de chiffrer l'importance des dégâts causés par le ragondin, de connaître la biologie de l'espèce, d'identifier ses maladies et parasites et de mesurer les effets de la lutte par piégeage et par empoisonnement. À la fin des années 1980, plusieurs thèses seront publiées sur ces sujets et ce travail en réseau débouchera en 1996 sur la publication d'un livre édité par l'ACTA : Le ragondin - Biologie et méthodes de limitation des populations, sous la direction de P. Jouventin, T. Micol, C. Verheyden. et G. Guédon et qui est décrit par certains comme «la bible» du ragondin. Une dynamique qui va cependant s'essouffler car au moment de la parution du livre, le noyau de ses auteurs apparaît éparpillé en raison de l'absence de politique de recherche sur le sujet par les organismes concernés et du manque de financement.

En 1994, le groupe «Rongeur» de l'Association nationale de la protection des plantes réunissant des partenaires privés et publics recommande une «étude prioritaire » devant permettre d'estimer les populations de rongeurs aquatiques. Dans ce même groupe, en 1996, on insiste sur le rôle indispensable de la recherche appliquée sur ce type de sujet. En 1998, un programme de recherche en collaboration avec le laboratoire CNRS de Chizé est proposé. Parallèlement, la Fédération nationale des GDEC propose un projet d'étude sur les impacts du ragondin, la traçabilité des luttes collectives, leur efficacité et la recherche de nouveaux moyens. En 1998 toujours, l'ACTA réunit différents partenaires sur le thème de la contraception immunologique et chimique. Les méthodes présentées seront jugées intéressantes mais nécessitant des recherches complémentaires.

Toutes ces recommandations débouchent finalement sur deux études : une expérimentation menée sur l'étang de l'Or (Hérault) réalisée par l'Inra de Montpellier et un travail portant sur la responsabilité du ragondin en matière de transmission de la leptospirose, confié à l'École vétérinaire de Nantes et qui sera réalisé avec la participation des membres des FDGDEC.

Actuellement, les gestionnaires des actions de lutte observent des populations de ragondins élevées, colonisant de nouveaux territoires comme la Bretagne, le Massif central, les contreforts des Pyrénées. Leurs interrogations sont d'autant plus fortes que le climat de ces dernières années est qualifié de clément.

Pourtant, les études sur le ragondin restent fragmentées, elles arrivent difficilement à être diffusées au-delà des terrains sur lesquels elles sont produites. Contre cette tendance, des structures comme le Forum des marais atlantiques et l'Entente interdépartementale de démoustication veulent réagir. Elles se heurtent cependant à divers problèmes. D'abord ces sujets d'études ne semblent plus réellement attractifs au sein des institutions de recherche. Aujourd'hui, la gestion du monde rural doit compter avec une mosaïque de structures très diversifiées (parcs, syndicats mixtes, fédérations et associations diverses, sans oublier les régions et départements, qui sont les principaux bailleurs de fonds). Et chacune de ces structures entend être ou rester le porte-parole des milieux qu'elle représente.

En France, la connexion entre réseaux de production des connaissances sur ces rongeurs envahissants et réseaux d'action apparaît comme un véritable défi.

sont à disposer sur des radeaux. Au fil des années, la bromadiolone a cependant perdu le statut d'une molécule au-dessus de tout soupçon. Est-elle réellement sans danger pour l'homme, pour les autres espèces animales ou pour la qualité de l'eau? Est-elle fabriquée dans de bonnes conditions, bien manipulée, bien appliquée? Ne pourrait-on lui substituer un autre produit plus sûr? Le risque de contamination et d'empoisonnement des chaînes alimentaires a en tout cas été prouvé dans le cas de la lutte contre le campagnol terrestre. Dans l'est de la France, des groupes de naturalistes ont en effet démontré que les actions de lutte chimique ont des conséquences indéniables sur la faune sauvage chassable, et plus particulièrement sur certaines espèces protégées. Qu'en est-il alors des effets de la lutte chimique contre le ragondin sur la loutre ou le campagnol aquatique? Dans un tout autre dossier, les controverses qui portent sur la qualité de l'eau en Bretagne apportent un surcroît d'incertitudes, sachant que les appâts empoisonnés sont censés être placés sur l'eau.

Pour ces différents motifs, mais aussi parce que l'Union européenne a placé les produits toxiques sous haute surveillance, en France, la lutte chimique est désormais en sursis ${ }^{5}$. Elle n'est plus autorisée que de façon temporaire, dans des conditions "très encadrées » et en complément à d'autres moyens d'action, c'est-àdire principalement le piégeage. Dans les différentes régions colonisées par le ragondin, des équipes de piégeurs ont ainsi été renforcées ou même créées. Ces nouvelles structures relèvent à chaque fois d'arrangements locaux et leurs modes d'organisation sont variables. Soit, elles recrutent des professionnels chargés de l'entretien des zones humides, soit ce sont des associations de réinsertion sociale, ou encore des réseaux de piégeurs bénévoles. À toutes ces personnes, il est proposé de capturer le ragondin au moyen de cages, une solution technique qui permet de garder la vie sauve aux espèces menacées (Encadré 3).

De nos entretiens, il ressort qu'il est impossible de parler du ragondin sans évoquer les actions de lutte menées contre lui. Et l'interdiction de l'empoisonnement,

\footnotetext{
${ }^{5}$ Décret $\mathrm{n}^{\circ}$ 2003-867 du 5 septembre 2003, arrêté du 8 juillet 2003.
} 
même si elle n'est pas encore d'application, remodèle en profondeur le paysage de ces actions. Nos interlocuteurs s'interrogent alors longuement sur leur pertinence, leurs choix techniques et leurs modes d'organisation. À travers leurs propos, nous découvrons des clivages profonds, sans doute prévisibles, mais aussi de nombreux petits partages, inédits. Ils montrent aussi la grande diversité des catégories de représentation qui s'y rattachent et se combinent les unes aux autres.

\section{Faut-il combattre le ragondin?}

Qu'est-ce qui justifie la lutte contre le ragondin ? Cette question semble être la base du problème et elle définit un clivage simple entre ceux pour qui la nécessité de ces actions est évidente et ceux qui les refusent de façon nette. Pour les premiers, la situation est claire : «Le ragondin fait partie des animaux avec lesquels on ne peut pas vivre. C'est comme les rats et les souris : c'est-àdire qu'on vit avec... mais par la force... » (responsable FDGDEC). Cette cause sans appel justifie les luttes menées sous leur forme actuelle: «Chez nous, le ragondin est un fléau et je pense que ce que nous faisons (i.e. mener des opérations d'empoisonnement) est bien » (responsable agricole). Mais face à cette opinion ferme, une autre évidence s'impose : «Les gens ne sont plus capables d'accepter la faune telle qu'elle est. Il faut toujours qu'ils en éliminent là... et qu'ils en rajoutent là... » (scientifique). Tout se passe alors comme si on assistait à une scène cent fois rejouée avec comme protagonistes ceux pour qui la nature doit s'aligner sur des critères d'utilité et de rentabilité et ceux pour qui la nature doit être préservée, soustraite à un excès d'interventions intempestives.

Pourtant, l'affaire est un peu plus compliquée que cela, car la présence du ragondin révèle aussi l'ambiguïté qu'entretiennent les gestionnaires de la nature avec les espèces invasives. «Le ragondin n'a pas sa place dans la faune française » nous affirme un scientifique, et cet autre souligne que suite aux introductions multipliées d'espèces non indigènes : «Certains espaces naturels protégés ne sont plus fonctionnels et il faut désormais une intervention humaine pour les sauver ». Et ceci justifie la lutte contre le ragondin (par piégeage) dans les espaces protégés. Mais à l'inverse, d'autres gestionnaires revendiquent pour cet animal le droit à se définir lui-même : «Le ragondin est là depuis longtemps, je ne dirais pas qu'il a une place, mais il l'a faite! » C'est donc son adoption pure et simple qui est alors proposée : «Le ragondin fait désormais partie de notre patrimoine. » Ces avis divergents recoupent ainsi deux définitions différentes qui sont données du sauvage à travers lesquelles s'exprime une recomposition mouvante de la nature et du rôle que l'humain peut ou veut y jouer. Pour les uns, l'animal sauvage est une espèce autochtone, d'autant plus digne d'intérêt qu'elle est menacée et qu'il faut la protéger, en particulier de la concurrence exercée par les espèces non indigènes. Mais pour les autres, un animal sauvage est avant tout un animal capable de mener une existence sans intervention humaine, spontanée, autonome.

\section{Quel sens donner aux actions de lutte?}

En France, le principe de la lutte obligatoire a été acquis de façon légale, mais ce n'est pas pour autant que celui-ci va donner à l'action un sens unanimement partagé. Ici s'ouvre un nouveau clivage entre ceux qui préféreraient «éradiquer » l'espèce et ceux qui entendent «gérer» ses populations. Dans la pratique, ce choix ne se pose pas vraiment parce que l'élimination pure et simple du ragondin paraît impossible. Pourtant dans les esprits, cette distinction reste présente et elle donne à la lutte un sens différent. Pour ceux qui voient avant tout cet animal comme un fléau et qui se présentent comme des victimes (principalement les agriculteurs), le ragondin doit être éliminé dans toute la mesure du possible. Mais d'un autre côté, l'accent qui est mis actuellement sur la lutte mécanique met aussi en scène des acteurs qui s'identifient d'abord en tant que chasseurs ou piégeurs et pour qui : «aucune espèce ne doit disparaître ».

La distinction éradication-gestion recoupe aussi de façon récurrente la question qui porte sur les moyens de lutte, principalement chimiques ou mécaniques. Comment trancher? À propos de la lutte chimique, un responsable d'association agricole affirme : «Il faut être inquiet, mais aussi réaliste, ce que nous faisons est bien... » Les effets de la bromadiolone dans la nature sont cependant de plus en plus mis en cause. Et un autre responsable agricole nous confie : «Il faut se mettre dans la tête que ce genre de produits, c'est fini, même si on n'a jamais eu de véritable accident. C'est comme pour le maïs, on a appliqué des produits non solubles dans l'eau et il faut pouvoir stopper avec ça... » Mais plus fondamentalement, la question est-elle seulement d'éviter des accidents ou de pouvoir encore justifier le recours à des produits chimiques? Certains expriment leurs hésitations, comme ce responsable d'action de lutte qui nous confie hors enregistrement : «En tout cas, je refuse l'étiquette d'empoisonneur. » Le choix entre le poison et le piège est un sujet sensible. Pour les agriculteurs qui se perçoivent comme des victimes, interdire la bromadiolone représente une expression supplémentaire de leur marginalisation au sein de la société. Pour d'autres, le choix de la méthode est fondamentalement en cause. Ils préfèrent renoncer purement et simplement à combattre le ragondin si la lutte chimique reste autorisée.

Mais la distinction éradication-gestion renvoie encore à la valorisation qui peut être attendue de la mort des animaux. D'un côté, certains craignent qu'elle ne rejaillisse sur l'efficacité des actions : "Quand on est dans une optique de valorisation, on s'aperçoit que tous les jeunes 
sont systématiquement relâchés, parce que les peaux ne sont pas commercialisables, qu'il faut les laisser grandir, qu'on relâche les femelles lorsqu'elles sont gestantes, etc. Donc, c'est une volonté qu'on a d'éviter de parler de valorisation » (responsable FDGDEC). Mais d'un autre côté, certaines personnes estiment que la valorisation des animaux morts donnerait une justification à ces actions. Ici s'exprime l'idée d'une nature intégrée aux activités humaines et qui donne sens à la vie autant qu'à la mort : «C'est vrai que si on entrait dans une logique d'exploitation, vente des peaux ou de la viande, on verrait ces animaux comme une ressource. Cela permettrait de les intégrer mieux dans la société, dans l'économie. Du coup, ils ne seraient plus un fléau contre lequel il faut lutter, mais ils feraient partie des ressources de la nature » (scientifique). Ici l'éradication rime systématiquement avec un refus de valorisation. En revanche, la gestion va quant à elle être combinée, ou non, avec la valorisation. Une valorisation qui, si elle était mise en œuvre, aurait encore à fournir les preuves de sa rentabilité...

\section{Y a-t-il une bonne mort pour un animal ?}

Quoi qu'il en soit, l'option du piégeage s'avère aujourd'hui celle qui est la plus généralement acceptée, mais les débats ne sont pas clos pour autant. Comment le mettre en œuvre et avec quels pièges? D'une façon qui n'est qu'apparemment paradoxale, certains piégeurs continuent à préférer les pièges tuants, car un animal pris dans une cage doit ensuite être mis à mort. Ce choix technique rappelle que dans la lutte chimique, les animaux empoisonnés se réfugient dans leurs terriers. Leur mort décalée dans le temps devient alors invisible.

Avec la généralisation de la cage s'ouvrent aussi de nouvelles questions : "Cette mise à mort, ça passe mal et souvent, ça se passe mal. Pour assommer le ragondin pris au piège, il faut être très adroit. Pour le tuer avec un fusil, il faut respecter les règlements et c'est mal vu. Finalement, beaucoup de gens le noient et ça dure trop longtemps. Il y a chez certains pas mal de sadisme, et en même temps, on ne peut pas parler de tout ça, notre société ne le supporte pas » (scientifique). En définitive, il semble que le piège parfait n'ait pas encore été découvert : «Vous savez, le super-truc, le miracle, ça n'existe pas. Un piège, ça peut aussi devenir du massacre, alors je veux bien qu'on critique le chimique mais... » (responsable FDGDEC).

Chacun à sa façon définit alors les limites de l'acceptable. Ainsi ce piégeur qui refuse catégoriquement l'empoisonnement : «l'animal meurt dans une agonie longue et abominable », mais qui se réjouit d'attraper d'un seul coup une femelle avec tous ses petits. Ou cet autre qui déclare : «Là où on piège (les nuisibles), on revoit la vie... » Et cette personne qui élève des ragondins en cage (production de pâté) et déclare : «Pour savoir combien il faut de poison en moyenne pour tuer un ragondin, ils font des expériences qui sont à vomir... ça rappelle Auschwitz... » La lutte contre le ragondin engage des controverses techniques sur les modes de piégeage, mais plus fondamentalement, celles-ci expriment des sentiments contrastés sur la mise à mort. Dans ces discussions, chacun compose alors la liste des animaux avec lesquels il accepte de vivre ou non. Et il définit pour eux les règles de la vie - d'une bonne vie -, mais aussi celles de la mort - d'une bonne mort.

\section{Comment qualifier le piégeage professionnel?}

En France, la lutte contre certains animaux proliférants (le ragondin et, dans le Nord, le rat musqué) est devenue un projet professionnel à part entière qui doit compléter les pratiques solitaires et plus traditionnelles des piégeurs bénévoles. C'est une nouvelle mission qui s'ajoute à celles des FDGDEC (qui coordonnent encore la distribution d'appâts empoisonnés). Mais le piégeage collectif est également organisé par des structures récemment créées. Dans ce cas, il est régulièrement conjugué avec d'autres tâches d'entretien de l'espace, notamment l'arrachage d'une espèce végétale envahissante, la jussie. Le plus souvent, on recrute pour ces tâches des jeunes ou des personnes en recherche de réinsertion sociale. Que dire du profil des agents engagés pour ce nouveau métier? Certains ont une "mentalité de chasseurs » et ils apprécient cette activité autonome et à l'extérieur. Il n'en reste pas moins que l'essentiel de leur travail consiste à parcourir des zones humides par tous les temps et à tuer de sang-froid des animaux, ce qui n'est pas forcément simple à faire ou même à dire.

Quand les moyens matériels des associations le permettent, ces nouveaux professionnels travaillent par deux et avec des petits véhicules $4 \times 4$ : « Avec les quads, c'est plus rigolo" (piégeur professionnel). Outre le fait que ceci leur confère un instrument de travail qui participe à leur identité, les quads leur évitent de porter, parfois sur de longues distances, les cadavres des animaux tués. Dans notre enquête, nous avons découvert que les nouveaux professionnels du piégeage appellent systématiquement le ragondin : « $\mathrm{ra}(\mathrm{t}) »$. En raccourcissant son nom, en assimilant ce gros rongeur placide aux rats, tout se passe comme si leur métier en devenait plus évident. Utiliser une qualification sans ambiguité permet de clarifier les choses, mais parfois aussi de masquer un désarroi latent (Porcher, 2003).

Certains encadrants sont en revanche plus explicitement perplexes sur cette question de mise à mort : "Cela me fait quelque chose, je ne me sens pas vraiment prêt à aider mes agents... » Mais, ils l'abordent aussi toujours en marge des entretiens : "Mon collègue dit que c'est un travail de boucher » lâche un chargé de mission, entre 
deux portes. Ces propos sont tenus debout, en se quittant, hors enregistreur ou encore sur un mode euphémisé, comme en témoigne l'anecdote qui suit. Nous sommes sur le terrain, en compagnie d'une chargée de mission, de deux piégeurs salariés et... de deux ragondins récemment attrapés dans une cage. Quand nous quittons les piégeurs, notre interlocutrice s'étonne qu'ils ne nous accompagnent pas : "Ah oui, vous devez encore... heu, heu, heu... » Mais au caractère feutré qui est généralement adopté pour évoquer la mise à mort des animaux, s'oppose l'interpellation forte qui nous a été lancée par cette dame, membre du parti des Verts et adjointe au maire dans une ville rurale : «C'est sûr que je suis contre la lutte chimique... même surveillée, il y a toujours des risques... Je suis donc vraiment pour le piégeage. Mais il faudrait que ce soit reconnu comme une vraie profession, avec un salaire convenable. Et en ce qui concerne les personnes à réinsérer, on pourrait leur proposer quelque chose de plus agréable que de tuer des animaux!»

Les cages utilisées par les nouveaux professionnels du piégeage apparaissent sans contestation comme le mode de régulation le moins nocif pour les espèces menacées. Mais elles imposent une mise à mort des animaux sans détour, ce qui, du coup, exige un supplément de justifications. Et de façon inattendue, le rejet de la lutte chimique conduit donc à interroger les conditions de ce nouveau travail et le statut de ceux à qui ces actions sont dévolues.

\section{Quel risque sanitaire ? Comment l'évaluer et en parler?}

En plus des dégâts aux cultures et aux berges des cours d'eau et canaux, une autre conséquence de la prolifération de ces animaux a trait au risque sanitaire qu'ils représentent en tant que mammifères porteurs de la leptospirose, de la toxoplasmose et d'autres maladies plus rares. Une étude récente a confirmé ce fait à propos de la leptospirose, maladie transmissible à l'homme (via l'urine, en milieu aquatique) qui, si elle n'est pas identifiée à temps, est mortelle. Du fait de leur très grand nombre et de leur attribut de nuisible, les ragondins se prêtent évidemment bien à de telles hypothèses, mais comme le remarque un gestionnaire de la faune sauvage : «Il n'y a aucune raison de penser que les espèces protégées ne soient pas elles aussi porteuses, le castor par exemple. En fait, on ne le sait pas et on n'a pas de matériel pour le dire... » L'étude menée sur les ragondins est-elle justifiée par le risque lié à la prolifération de ces animaux ou, comme le demandent certains, constitue-t-elle un argument supplémentaire pour légitimer les actions de lutte contre eux?

Ces questions appellent aussi des réponses ambivalentes. Dans leurs propos, certaines personnes mettent en effet le risque sanitaire fortement en évidence. Ce risque justifie et renforce les actions de lutte. Pourtant, l'ombre du syndrome «vache folle » plane désormais sur les réflexions et laisse pressentir un autre risque : «Il ne faut pas trop en rajouter, ne pas susciter une trop grande inquiétude [...] mettre en branle des moyens qui seraient disproportionnés » (scientifique). Et le côté machiavélique d'une opération de communication de grande envergure n'échappe pas à certains responsables, conscients du fait que certains messages peuvent être très, voire même trop, faciles à manipuler : «Vous créez de la psychose et ça marcherait à tous les coups. Si vous insistiez sur les risques non seulement pour l'homme mais aussi pour les animaux domestiques, les chiens par exemple, on aurait tous les droits pour exterminer le ragondin. On pourrait à nouveau parler de poison alors! On vous pendrait haut et court, si vous n'en mettiez pas! (secrétaire d'une fédération régionale de chasse).

Adopter une voie moyenne entre dramatiser et ne rien dire suppose un choix difficile, parce que les actions de prévention devraient être adaptées à des publics spécifiques : "Aujourd'hui, avec les activités de loisirs qui ne cessent d'augmenter, le risque sanitaire est plus important et les gens n'ont pas du tout conscience de cela. Mais leurs perceptions sont très différentes : on ne peut pas passer la même information en ville et en zone rurale, il y a vraiment deux univers... En ville, les gens trouvent le ragondin sympathique, alors qu'à la campagne, les personnes sont d'abord préoccupées par leurs problèmes qui sont à $99 \%$ de type rural » (scientifique). La représentation de ces rongeurs est donc aussi reliée au risque de maladie, mais lequel? Comment le réduire? En parler? Et comment faire de la prévention auprès des piégeurs et des professionnels (ce qui paraît assez facile) et auprès de tous les usagers des rivières et plans d'eaux (ce qui s'avère beaucoup plus compliqué)?

\section{Quel territoire partager?}

Un dernier aspect, mais non le moindre, consiste à observer que, pour nos interlocuteurs, parler du ragondin, c'est inévitablement évoquer un territoire et son histoire, ses habitants ou ses usagers. En Camargue, la lutte contre le ragondin est vue comme "une porte d'entrée » pour évoquer la gestion collective des niveaux d'eaux, qui se heurte à une privatisation forte de l'espace. La lutte devient le prétexte pour parler d'un équilibre naturel difficilement imaginable : «On préconise l'à sec annuel en juillet, août. On voudrait faire adhérer les propriétaires à cette gestion et ainsi les actions de piégeage devraient diminuer » (gestionnaire d'espace naturel). Dans le Marais poitevin, les actions sont liées à la promotion d'un corps professionnel d'agents d'entretien de l'espace : «On veut mettre en évidence la nécessité de développer des emplois sur le marais pour couvrir des missions d'utilité collective, anciennement couvertes par les propriétaires privés, l'entretien du paysage, pour que les touristes 
puissent voir un marais tel qu'il est sur les cartes postales [...] D'une certaine façon, on peut dire que l'histoire du ragondin, c'est aussi celle du parc naturel régional... » (gestionnaire d'espace naturel). À l'opposé, dans le Marais breton, le choix qui s'est imposé consiste à mettre en place un réseau de piégeurs bénévoles, composé d'habitants : «Ici il y a un attachement fort des gens au marais. On aurait des problèmes, je pense, de droit d'accès aux parcelles, si on avait des professionnels... Les gens ici disent qu'ils n'ont besoin de personne, qu'ils piègent déjà chez eux depuis longtemps... Le Marais breton, c'est essentiellement de l'élevage, le marais est incultivable. Dans le Marais poitevin, ce n'est pas la même agriculture, ce sont des champs ouverts, des céréaliers... ils ne vont pas passer leur temps à aller piéger... » (chargé de mission). Mais les exemples ne s'arrêtent pas là, car dans le Marais poitevin, la représentation du ragondin comme fléau est aussi reliée à la PAC qui a favorisé l'assèchement et l'intensification agricole. Et en Bretagne, c'est la mauvaise qualité des eaux de surface qui a conduit d'emblée à refuser la lutte chimique, etc.

Zones humides pour lesquelles on cherche à retrouver un certain équilibre naturel, zones de marais à gérer de façon experte pour des usagers locaux ou non, ou encore zones de marais à gérer entre soi... Dans les milieux où nous avons enquêté, l'organisation des actions menées contre le ragondin devient ainsi une occasion pour profiler l'avenir d'un territoire, faire des recommandations ou simplement pour justifier que les choses restent comme elles ont toujours été.

\section{Le ragondin est un animal envahissant}

Le ragondin est un animal insaisissable, au sens propre, c'est-à-dire dans l'espace, et pour nous aussi, au sens figuré. Cette difficulté est devenue une question centrale dans notre étude. En nous basant sur les réflexions des personnes que nous avons rencontrées, sur leurs connaissances d'origines diverses et qui leur permettent d'encoder l'histoire et les expériences (Fradin et al., 1994), nous avons cherché à explorer la diversité des catégories qui définissent cet animal et les différentes façons de percevoir les actions menées contre lui. Car celles-ci supposent des formes d'organisations et des choix techniques qui, en retour, ont des impacts sur la nature et les hommes (Roussel, 2003; Mougenot et Roussel, 2005). Notre enquête montre alors qu'il n'y a pas de clivages simples entre humains ni espèces, mais de nombreuses catégories qui correspondent à des lignes de partage, fortement contextualisées, inscrites dans des lieux, des moments et liées à des activités. Et en même temps, ces représentations sont confrontées à de nouveaux problèmes, elles sont de ce fait interdépendantes, susceptibles de changer rapidement ou de s'assembler les unes aux autres pour des motifs parfois très différents.
L'histoire de l'introduction du ragondin en Europe et du traitement qui lui a été réservé montre ainsi que «ce qui compte» pour nous, humains, n'a rien de définitif, ni de communément partagé, et elle souligne nos rapports diversifiés et toujours changeants à la nature. Nous avons ainsi découvert cet animal comme le héros d'une fable postmoderne, qui nous parle de nos difficultés à construire des catégories stables pour la réflexion autant que pour l'action. Et la morale de cette histoire est qu'aujourd'hui, il s'agirait d'aligner de manière satisfaisante le refus de dégâts aux cultures et aux infrastructures avec l'identité des habitants de chaque territoire, avec la qualité d'un travail à proposer à ceux qui n'en ont pas, avec un risque sanitaire réduit, avec une gestion experte de l'espace, avec la nécessité d'infliger une mort sans souffrance aux animaux à supprimer, avec le plaisir de découvrir encore la nature en ville, avec une protection accrue des espèces menacées, avec...

Depuis qu'il a été imposé au ragondin de quitter ses frontières naturelles, tout se passe comme s'il n'avait cessé d'en franchir d'autres, c'est-à-dire d'emmêler les catégories physiques, conceptuelles, symboliques ou pratiques... Décidément, il s'agit bien là d'un animal très envahissant...

\section{Références}

Chaigneau, A., 1967. Animaux nuisibles à la chasse - mammiferes - rapaces, Paris, La maison rustique.

Despret, V., 2002. Quand le loup habitera avec l'agneau, Paris, Les empêcheurs de tourner en rond.

Fradin, B., Quéré, L., Widmer, J., 1994. L'enquête sur les catégories. De Durkheim à Sacks, Raisons Pratiques, Paris, École des Hautes Études en Sciences Sociales.

Gosling, M., 1989. Extinction to order, New Scientist, 4, 44-49.

Jouventin, P., Micol, T., Verheyden, C., Guedon, G., 1996. Le Ragondin, Biologie et méthodes de limitation des populations, Paris, Association de coordination technique agricole.

Maurice, A., 1931. Le ragondin, Paris, Archives d'Histoire Naturelle de la Société d'Acclimatation.

Melard, F., 2001. L'autorité des instruments dans la production du lien social, le cas de l'analyse polarimétrique dans l'industrie sucrière belge. Thèse pour l'obtention du grade de docteur, Ecole des Mines, Paris.

Mougenot, C., 2003. Prendre soin de la nature ordinaire, Paris, Maison des Sciences de l'Homme et INRA.

Mougenot, C., Roussel, L., 2005. To poison or to Trap? The Ecologisation of 'Pest' Control, Sociologia Ruralis, 45, 1-2, 115-129.

Porcher, J., 2003. La mort n'est pas notre métier, Paris, éd. de l'Aube.

Roussel, L., 2003. From poisoning to trapping: some thoughts about muskrat and coypu control in France, 4th European Pest Management Conference. Parma, Italie, 9-12 septembre.

Sheail, J., 2003. Government and the Management of an Alien Pest Species: a British Perspective, Landscape Research, 28, 1, 101-111. 